\title{
Protein Expression via the Molecular Chaperone ClpL
}

\author{
Hyog Young Kwon ${ }^{1 \#, ~ S a n g-S a n g ~ P a r k ~}{ }^{2 \#, ~ M o h a m m a d ~ F a r i d ~ Z i a 1 ~ a n d ~ D o n g-K w o n ~ R h e e ~}{ }^{2^{*}}$ \\ ${ }^{1}$ Soonchunhyang Institute of Medi-bio Science, Soonchunhyang University, Cheonan 31151, Republic of Korea \\ ${ }^{2}$ School of Pharmacy, Sungkyunkwan University, Su-Won 16419, Republic of Korea \\ "These authors contributed equally to this work
}

\begin{abstract}
Protein expression using bacterial systems has advanced substantially over the past few decades, but Escherichia coli is still the most commonly utilized expression host, despite issues related to protein solubility. Several solutions, such as different host strains, different vectors, and incubation with co-chaperones, have been developed to minimize protein aggregation and ensure high-quality protein production. Here, we review commonly used methods to increase protein solubility, with a focus on the Clp/Hsp100 family and pneumococcal ClpL, a novel member of the Clp/Hsp100 family that is highly induced in Streptococcus pneumoniae during heat shock. Unlike the DnaK system, which requires an additional co-chaperone system to reinstate the natural conformation of denatured target proteins, pneumococcal ClpL is able to disaggregate denatured proteins independently, without requiring a co-chaperone system. Accordingly, ClpL could be a useful chaperone system to solubilize foreign proteins during protein overexpression.
\end{abstract}

Keywords: Protein aggregation; ClpL; Chaperone; Recombinant protein expression; Inclusion bodies

\section{Introduction}

Many systems are used to produce heterologous proteins in bacteria, but Escherichia coli is frequently used since it is easy to manipulate and has a short life cycle. Additionally, extensive genetic tools have been developed to facilitate the production of recombinant proteins. It is also easy to scale-up and costs relatively little to culture compared to other systems. Thus, $E$. coli has been the first choice for the production of recombinant proteins [1]. However, the use of E. coli as a host has a few limitations: 1) recombinant proteins may fail to form an appropriate conformational structure and 2) proteins that are produced in a large quantity tend to aggregate to form an insoluble inclusion body, which lacks structure and function. Although there are no universal solutions for these problems, many different approaches have been developed and these methods have substantially improved protein production. Methods for the production of high-quality, high-quantity proteins have largely been established by trial and error by changing various parameters, such as host strain, expression vector, protein sequences (without affecting the functional domain), chemical additions, and incubation with chaperones. Recently, while characterizing heat shock proteins (HSPs) of Streptococcus pneumoniae, we found that pneumococcal ClpL, a member of the Clp/Hsp100 family and a ClpB homologue of E. coli, is able to disaggregate denatured proteins without co-chaperone systems, such as the DnaK system. In this review, we summarize several strategies commonly used to increase protein solubility with a focus on the effects of incubation with chaperones, especially pneumococcal ClpL, to improve protein production. Thus, we suggest that it is highly feasible to produce recombinant proteins in E. coli by co-expressing ClpL.

\section{Strategies for recombinant protein expression}

Vector systems: Since recombinant technologies have emerged, many different types of vectors have been introduced in the market. When a recombinant protein is induced in high quantities with a strong promoter or a high concentration of an inducer, it tends to become degraded or to aggregate to form an inclusion body, and accordingly it does not regain its original conformation. It is important to choose an appropriate vector system to overexpress a recombinant protein. The promoter and fusion tag appear to be the most important factors when selecting a vector system because they affect the solubilization of the recombinant protein. One of the most commonly used vector systems is the pET vector from Novagen (Madison, WI, USA) and Clontech (Mountain View, CA, USA) because it contains a His-tag to enable the easy isolation of target proteins using a nickel column. The His-tag is relatively small compared to other fusion proteins, such as glutathione$S$-transferase (GST) or maltose-binding protein (MBP); accordingly, it is less likely to affect the function of a target protein. However, when a target protein aggregates and forms inclusion bodies, the target gene can be cloned in other vectors containing fusion proteins, e.g., GST [2], MBP [3], NusA [4], Sumo [5], and Trx [6]. Representative vectors include the pGEX system produced by GE Healthcare (Little Chalfont, $\mathrm{UK}$ ) and the pMal system produced by New England Biolabs (Ipswich, MA, USA). Fusion proteins are somewhat large and may interfere with the activity of target proteins if the tagging protein is not removed from recombinant proteins. Thus, multiple enzyme sites have been incorporated into vectors to facilitate the cleavage of fusion tags after overexpression and purification. Proteases that are commonly used include enterokinase (cleavage site DDDDK $\downarrow$ [7], factor Xa (cleavage site IEGR $\downarrow$ ) [8], SUMO protease (C-terminal end of the conserved GlyGly sequence in SUMO) [5], and thrombin (cleavage site LVPR $\downarrow$ GS) [9-11].

Host strains: Many different E. coli strains are available that are genetically manipulated to produce target proteins. For example, E. coli BL21 and its derivatives are widely used. This strain has deletions of the Lon and OmpT proteases; therefore, heterologous proteins can be expressed more stably [12]. Additionally, its derivatives, including BL21 (DE3) pLys S and BL21 (DE3) pLys E, have a lysozyme that is an

*Corresponding author: Dong-Kwon Rhee, School of Pharmacy, Sungkyunkwan University, Su-Won 16419, Republic of Korea, Tel: +82 2-760-0114; E-mail: dkrhee@skku.edu

Received January 23, 2016; Accepted February 06, 2016; Published February 13,2016

Citation: Kwon HY, Park SS, Zia MF, Rhee DK (2016) Protein Expression via the Molecular Chaperone ClpL. J Microb Biochem Technol 8: 076-082. doi: 10.4172/1948-5948.1000266

Copyright: ( 2016 Kwon HY, et al. This is an open-access article distributed under the terms of the Creative Commons Attribution License, which permits unrestricted use, distribution, and reproduction in any medium, provided the original author and source are credited. 
inhibitor of $\mathrm{T} 7$ polymerase. Thus, leaky expression of $\mathrm{T} 7$ polymerase in the strains is prevented by the lysozyme, resulting in higher host cell survival and increased target protein production [13]. As codons of target genes are often not present in the host, E. coli hosts are not able to produce target proteins. To resolve this issue, E. coli hosts, such as CodonPlus E. coli developed by Stratagene (La Jolla, CA, USA) and the Rosetta strain developed by Novagene have extra tRNA codons (AUA, AGG, AGA, CUA, CCC and GGA) [14,15]. When E. coli hosts produce target proteins at a high level, inclusion bodies tend to form. Reducing the incubation temperature helps bacteria produce more soluble proteins, but chaperone activity decreases drastically at lower temperatures [16]. To address this issue, the ArcticExpress E. coli strain developed by Agilent Technologies (Santa Clara, CA, USA) has a modified chaperone, which has protein folding activity at $4-10^{\circ} \mathrm{C}$ [17].

Chaperone co-expression: Heterologous proteins sometimes fail to reach their proper structural conformation, especially when the protein concentration in the cytoplasm of E. coli is too high. When the macromolecular concentration exceeds $300-400 \mathrm{mg} / \mathrm{ml}[18,19]$, appropriate protein folding is remarkably challenging. Chaperones help nascent polypeptide chains form their native conformations (Figure 1). In a crowded environment, the availability of chaperones might be limited and over-titrated, leading to protein instability and aggregation $[20,21]$. Thus, the addition of individual chaperones or chaperone sets may boost the availability of chaperones and help solubilize target proteins during overexpression $[22,23]$. The chaperone systems that are commonly used in E. coli are the DnaK, GroELS, and Clp/Hsp100 systems (Figure 1); commercially available chaperones are listed in Table 1.

\section{DnaK system and GroELS}

Living organisms face hostile environments, such as oxidative stress, nutrient starvation, and heat shock, and they cope with these environments by inducing stress-related proteins. HSPs are a group of well-studied stress proteins involved in many cellular processes, including cell division, translocation, thermo-tolerance, oxidative resistance, and chemical stress resistance. In prokaryotes and eukaryotes, many different types of HSPs have been identified; they have similar functions and molecular weights. HSPs are generally classified into DnaK, GroELS, Clp/HSP100, HtpG or small HSPs in prokaryotes depending on their molecular weights. The DnaK system, consisting of the DnaK (HSP 70), DnaJ (HSP 40), and GrpE (NEFs; nucleotide exchange factors), plays essential roles in protein folding and environmental stress resistance [24]. An important aspect of the DnaK system is that it works together with the ClpB chaperone, a member of the Clp/HSP100 family, during protein disaggregation (Figure 1) [25]. GroEL is an oligomer of over $800 \mathrm{kDa}$ that is assembled as two homoheptameric rings. One ring of GroEL possesses chaperone activity to fold misfolded or nonnative proteins via ATP hydrolysis with or without co-chaperonin [26-28].

\section{The Clp/HSp100 family of the AAA+ ATPase superfamily}

Caseinolytic protease (Clp)/heat shock protein 100 belongs to the $\mathrm{AAA}+$ (․TPase associated with various cellular activities) superfamily. This group of proteins refolds denatured proteins into their native form, acting as chaperones, and is also involved in the proteolysis of unnatural forms of proteins, thereby decreasing damaged and denatured proteins in an ATP-dependent manner [29]. Clp/HSP100 family proteins contain an N-terminal domain and one or two AAA+ nucleotide-binding domains (NBD1 and NBD2) separated by coiledcoil of alpha-helices middle domains (M-domain). The Clp/Hsp100 proteins are classified into two groups depending on the number of NBDs [30]. Class I proteins include ClpA, B, C, D, E, and L and contain two NBDs (NBD1 and NBD2). Class II proteins, such as ClpM, N, X, and Y, have only one NBD. The NBDs are responsible for ATP binding and hydrolysis.

The $\mathrm{Clp} / \mathrm{Hsp} 100$ protein family is involved in protein disaggregation and protein degradation and quality control [30]. ClpB/Hsp104 and $\mathrm{ClpL}$ mediate protein disaggregation, whereas other $\mathrm{Clp} / \mathrm{Hsp} 100$ proteins, including $\mathrm{ClpA}$ and $\mathrm{X}$, promote protein degradation in collaboration with the ClpP protease [30-32]. In the Clp/Hsp100 family, the $\mathrm{M}$-domain influences many activities, including ATPase activity and disaggregation as well as interactions with co-chaperones, such as DnaK, Hsp70, and MecA $[33,34]$. Though some Clp/Hsp100 proteins, including $\mathrm{ClpB}, \mathrm{E}$, and $\mathrm{L}$, contain an $\mathrm{M}$ domain, $\mathrm{ClpC}$ and $\mathrm{ClpA}$ have a shorter $\mathrm{M}$ domain and no $\mathrm{M}$ domain, respectively. Additionally, the sequence of the $\mathrm{M}$ domain in the Clp/Hsp100 family varies considerably. The importance of the $\mathrm{M}$ domain is well described for the mutant form of ClpB. When the $\mathrm{M}$ domain is deleted, ClpB loses its disaggregation activity, resulting in impaired thermo-tolerance $[35,36]$.

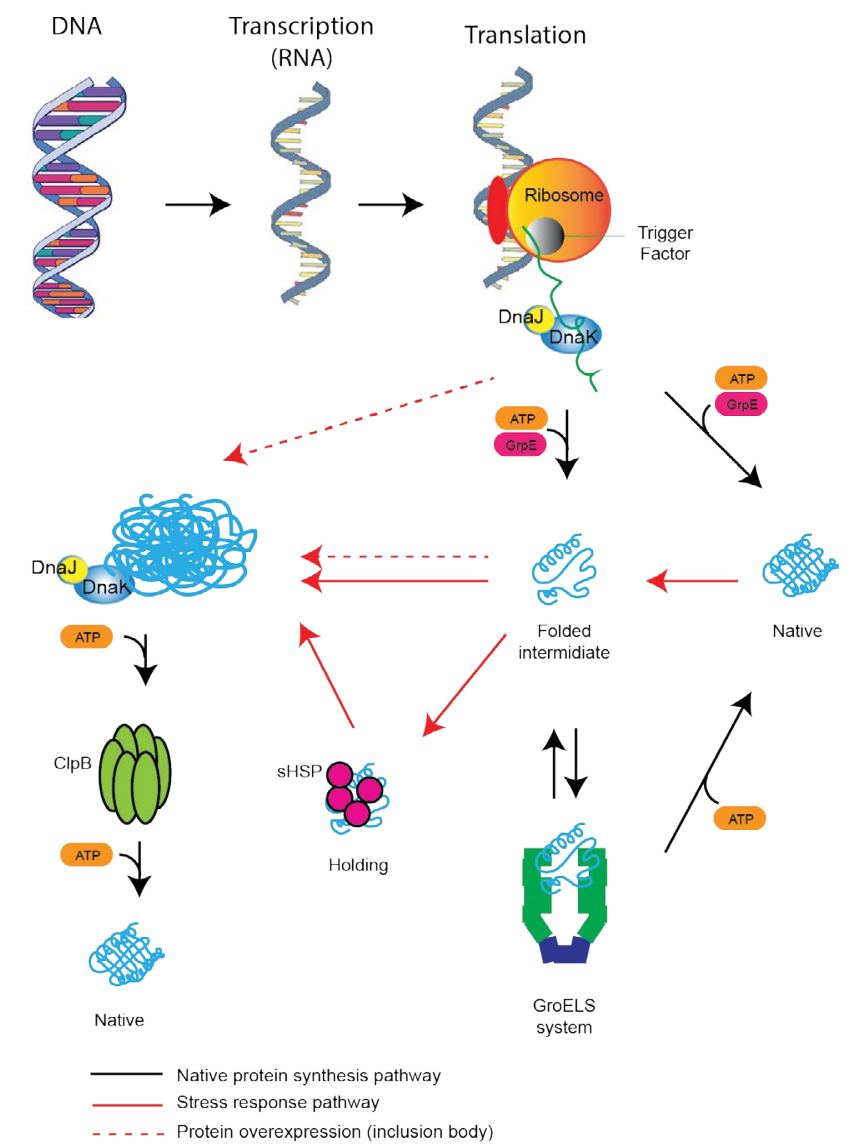

Figure 1: Chaperone-mediated protein refolding in Escherichia coli.

Nascent polypeptides fold into native proteins spontaneously or require assistance from other-chaperones, such as the DnaK system or GroELS system. The DnaK system, composed of DnaK, DnaJ, and GrpE (nucleotide elongation factor), interacts with $\mathrm{ClpB}$, a member of the Clp/HSP100 family, to reinitiate unfolded proteins. The GroELS system also folds proteins in intermediate states of folding. In the case of protein overexpression (red dotted lines) or stress conditions (red lines), the capacity of chaperones, such as the DnaK system or GroELS, to unfold misfolded proteins is limited, leading to protein aggregation or misfolding 


\begin{tabular}{|c|c|c|c|}
\hline Name & Chaperone & Promoter & Company \\
\hline pG-KJE8 & DnaK, DnaJ, GrpE (DnaK system), GroELS & araB and Pzt-1 & Takara \\
\hline pGro7 & GroELS & araB & Takara \\
\hline pKJE7 & DnaK, DnaJ, GrpE (DnaK system) & araB & Takara \\
\hline pG-Tf2 & Tig (trigger factor), GroELS & Pzt-1 & Takara \\
\hline pTf16 & Tig (trigger factor) & araB & Takara \\
\hline pBB530 & GrpE (DnaK system) & $\mathrm{PA} 1 / \mathrm{lacO} 1$ & Addgene \\
\hline pBB535 & DnaK, DnaJ (DnaK system) & $\mathrm{PA} 1 / \mathrm{lacO} 1$ & Addgene \\
\hline pBB540 & GrpE (DnaK system), ClpB (HSP 100 family) & $\mathrm{PA} 1 / \mathrm{lacO} 1$ & Addgene \\
\hline pBB542 & DnaK, DnaJ, GroESL(Large amounts) & $\mathrm{PA} 1 / \mathrm{lacO} 1$ & Addgene \\
\hline pBB550 & DnaK, DnaJ, GroESL(Small amounts) & Plac/lacO1 & Addgene \\
\hline pBB872 & ibpB, ibpA (small HSPs) & PA1/lacO1 & Addgene \\
\hline pColdl-IV & None (Cold shock protein promoter) & CspA & Takara \\
\hline
\end{tabular}

Table 1: Chaperone co-expression systems.

Partial deletions or point mutations of the $\mathrm{M}$ domain in $\mathrm{ClpB}$ result in a similar or less stringent phenotype as compared to the complete deletion [36-40]. However, unlike bacterial ClpB and yeast HSP104, even though ClpA does not contain an $\mathrm{M}$ domain, it can disaggregate proteins without a co-chaperone system, such as the DnaK system, suggesting that disaggregation reactions do not require the DnaK system [41].

The function of Clp/Hsp100 proteins is modulated by a group of proteins termed adaptor proteins, which are required for chaperone activity as well as substrate proteolysis [42]. Examples of adaptor protein are $\mathrm{MecA}, \mathrm{ClpS}, \mathrm{YpbH}$, etc. $\mathrm{ClpC}$ and $\mathrm{ClpA}$ require the adaptor proteins MecA and ClpS, respectively, for chaperone activity [43]. Adaptor protein ClpS in E. coli ushers substrate proteins to ClpAP for degradation. ClpA is positioned at either or both ends of the proteolytic core, where it modulates substrates to enter the catalytic chamber of ClpP through the narrow central pore of ClpA [32].

\section{ClpL of Streptococcus pneumoniae}

$\mathrm{Clp} / \mathrm{Hsp} 100$ proteins are found in a wide range of taxa; ClpA is found in gram-negative eubacteria, such as E. coli, $\mathrm{ClpB}$ is found in most eubacteria and eukaryotes, $\mathrm{ClpC}$ is found in cyanobacteria, plants, and most gram-positive eubacteria, ClpD is found exclusively in plants, and ClpE is found in certain gram-positive eubacteria [44]. S. pneumoniae, a causative agent of otitis media, pneumonia, and meningitis, encounters heat stress conditions and undergoes heat shock responses when it penetrates, colonizes, and invades the blood and brain. S. pneumoniae induces an array of heat shock proteins, such as the DnaK system, GroELS, and Clp/Hsp100, to cope with these hostile environments. These HSPs are highly conserved in both prokaryotes and eukaryotes, and are utilized against other environmental stresses, such as exposure to ethanol, antibiotics, and heavy metals, to enhance the survival rate of bacteria. The Clp/Hsp100 class chaperones of $S$. pneumoniae are ClpC, E, L and X [45], and an ATP-dependent protease of $S$. pneumoniae is ClpP, which plays an important role in in stress responses, competence, and virulence [46]. ClpC has several functions, such as the control of growth inhibition at high temperatures, autolysis, adherence, and transformation [47], and ClpE is required for growth at high temperatures and virulence [48]. ClpX is crucial for bacterial survival [45].

Previously, we indicated that ClpL is one of four major HSPs in S. pneumoniae that is induced by heat shock and mediates thermotolerance and protein disaggregation $[31,45,49,50]$. ClpL homologs have also been identified in many gram-positive pathogens, including Staphylococcus aureus, Listeria monocytogenes, Streptococcus pyogenes,
S. agalactiae, S. mutans, S. sanguinis, and the trypanosomatid protozoa responsible for leishmaniasis (Leishmania major) as well as lactic acid bacteria [31]. We and other groups have found that recombinant ClpL can refold denatured rhodanese and CtsR [49,51]. ClpL mutants are susceptible to penicillin and show thinner cell walls than the wildtype strains. Conversely, a pneumococcal strain overexpressing ClpL develops higher resistance to penicillin and a thicker cell wall. Consistent with this finding, when $S$. pneumoniae is exposed to penicillin, the bacteria exhibits upregulation of $\mathrm{ClpL}$ and penicillin binding protein 2 ( $\mathrm{Pbp} 2 \mathrm{X})$, a major cell wall synthesis enzyme, which improves survival in extreme conditions [50]. Recently, an antibiotic acyldepsipeptide (ADEP) activates ClpP, which results in complete eradication of $S$. aureus in combination with other antibiotics [52]. Likewise, ClpL in $S$. pneumoniae might be a highly promising target for antibiotic therapy.

\section{ClpL functions as a molecular chaperone without a co- operative chaperone system}

Pneumococcal ClpL and ClpP are highly induced after heat shock and are implicated in the regulation of a wide array of virulenceassociated genes, including $\operatorname{cbp} A, \operatorname{cps} 2 A$, ply, and $p s a A$ [49]. Although ClpL homologs are present in several different organisms, they have not been characterized at the molecular and biochemical levels. Recently, we conducted a biochemical study of ClpL of S. pneumoniae and found several interesting characteristics, which are distinct from those of other members of the Clp/Hsp100 family [31,50,53]. Recombinant ClpL displayed several functions, including nucleotide hydrolysis, refolding, holdase activity, and disaggregation, and used either $\mathrm{Mg}^{2+}$ or $\mathrm{Mn}^{2+}$ as a cofactor for optimal activity. $\mathrm{Mg}^{2+}$ and $\mathrm{Mn}^{2+}$ are divalent cations that are commonly found in biological systems, and $\mathrm{Mg}^{2+}$ is mainly utilized by AAA+ ATPase chaperones. For example, ATPase activity of ClpB, the closest $E$. coli homologue of $\mathrm{ClpL}$, is enhanced by $\mathrm{Mg}^{2+}$, but not by $\mathrm{Mn}^{2+}$. In the case of E. coli ClpA, $\mathrm{Mn}^{2+}$ actually inhibits ATPase activity. However, in other systems, such as eukaryotic Hsp104 and the GroELS system of E. coli, the activity is enhanced in the presence of $\mathrm{Mn}^{2+}$ [54]. Thus, although ClpL of S. pneumoniae belongs to the bacterial Clp/ Hsp100 family, it is unique because ATPase activity is enhanced by $\mathrm{Mn}^{2+}[31]$

Interestingly, $\mathrm{ClpL}$ does not require co-chaperones, such as the DnaK system, for chaperone activity and hexamerization (Figure 2). In contrast, $\mathrm{ClpB}$ requires the DnaK system for chaperone activity, and $\mathrm{ClpA}$ and $\mathrm{ClpC}$ collaborate with ClpP to provide protease activity [32]. However, pneumococcal ClpL does not collaborate with other co-chaperone systems or $\mathrm{ClpP}$; it has the capacity to disaggregate denatured proteins without co-chaperones and does not have a ClpP binding loop, a domain that is known to mediate the interaction 
Citation: Kwon HY, Park SS, Zia MF, Rhee DK (2016) Protein Expression via the Molecular Chaperone ClpL. J Microb Biochem Technol 8: 076-082. doi: 10.4172/1948-5948.1000266

(a) ClpB/ DnaKJ system
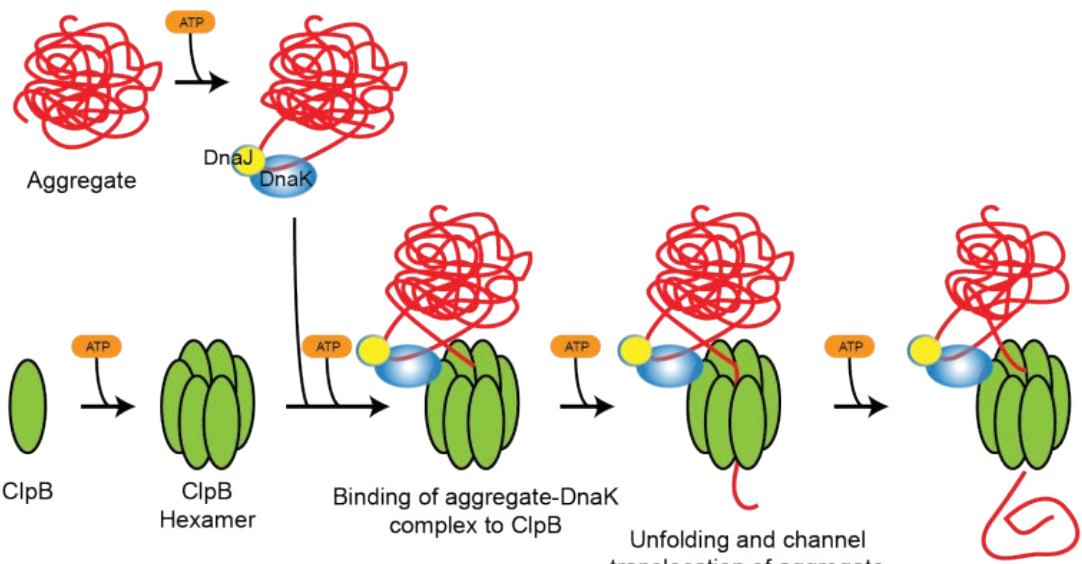

translocation of aggregate by ClpB

(b) ClpL system

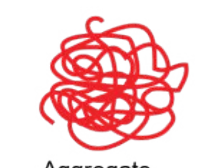

Aggregate

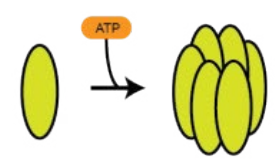

ClpL
ClpL

Hexamer complex to $\mathrm{ClpB}$
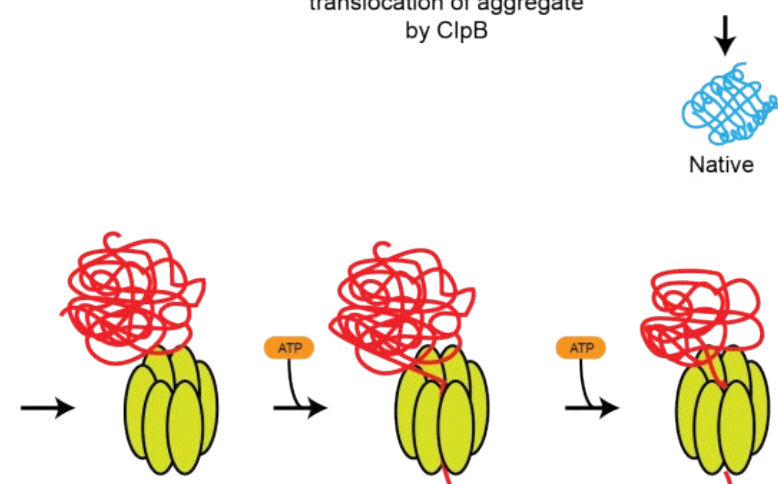

Binding of aggregate to

$\mathrm{ClpL}$

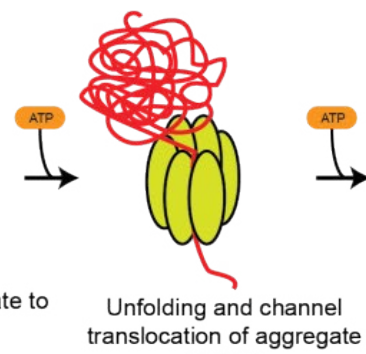

by ClpL

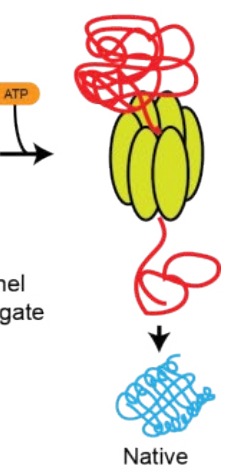

Figure 2: The difference between $\mathrm{ClpB}$ and $\mathrm{ClpL}$ in chaperone-mediated protein folding.

(a) ClpB requires the DnaKJ system to unfold aggregated proteins. The DnaKJ system initially binds to aggregated proteins and then ClpB interacts with the unfolded protein, resulting in protein disaggregation via ATP hydrolysis.

(b) ClpL does not require an additional co-chaperone system to refold protein aggregates. ClpL directly interacts with aggregated proteins to refold proteins into their native forms using ATP.

between Clp chaperone and protease complex [55]. Using Clustal Omega (http://www.uniprot.org/align/), we compared the protein sequences of the Clp/Hsp100 family to identify differences between ClpL and other family members (Figure 3). Clp/Hsp100 family proteins contain an $\mathrm{N}$-terminal domain, NBDs, and an M-domain, which mediates the interaction with auxiliary factors, such as DnaK, Hsp70, and MecA. Specific function of each domain in Clp/Hsp100 family is summarized in Table 2. M-domain of ClpC and ClpB mediates interaction with co-chaperone or auxiliary factors (ClpB: DnaK and ClpC: $\mathrm{MecA}$ ), but the M-domain of ClpL is much shorter than those of Hsp104 and ClpB, indicating that the M-domain of ClpL may have different functions from those of other $\mathrm{Clp} / \mathrm{Hsp} 100$ family members [31]. The N-terminal domain of $\mathrm{ClpA}$ and $\mathrm{ClpC}$ mediates interaction with auxiliary factors such as $\mathrm{ClpS}$ and $\mathrm{MecA}$, whereas that of $\mathrm{ClpB}$ interacts with the aggregated proteins (Table 2). Mutational analysis at the Walker A motifs (K127A/T128A and K458A/T459A) revealed that both NBDs are essential for chaperone activity, ATP hydrolase activity, and hexamerization.

Currently, ClpL has not been used as a model for optimization of protein expression in E. coli or other systems. However, as recombinant ClpL is known to refold denatured rhodanese and CtsR [49,51]. Thus, it is highly feasible that ClpL may become a good system for protein expression. Although ClpL is found in S. pneumoniae, S. pneumoniae per se would not be an ideal host since it is difficult to carry out transformation experiment due to thick capsule as well as rapid induction of autolysis when it reaches stationary phase. It might be required to generate mutants of genes involved in capsule synthesis or autolysis prior to exploitation of S. pneumoniae as a host.

\section{Conclusion}

Many systems for heterologous protein overexpression and 
Citation: Kwon HY, Park SS, Zia MF, Rhee DK (2016) Protein Expression via the Molecular Chaperone ClpL. J Microb Biochem Technol 8: 076-082. doi: 10.4172/1948-5948.1000266

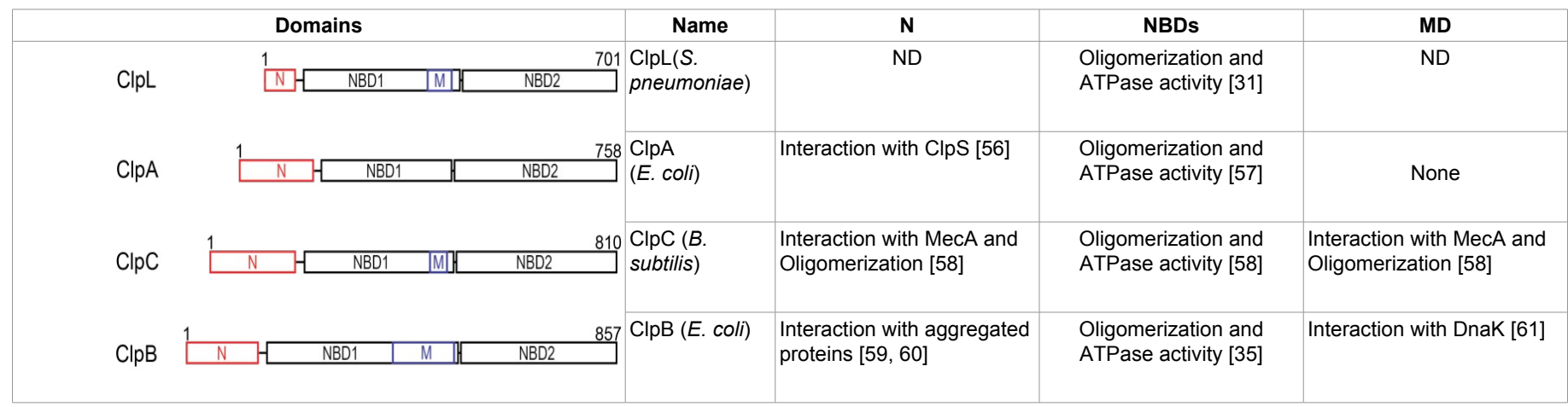

N: N-terminal domain, NBDs: Nucleotide Binding Domains, MD: Middle Domain, ND: Not Determined

Table 2: The domains of HSP 100 families.

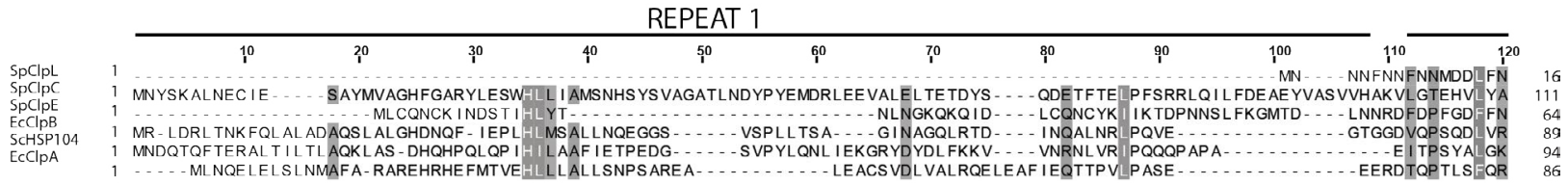

REPEAT 2
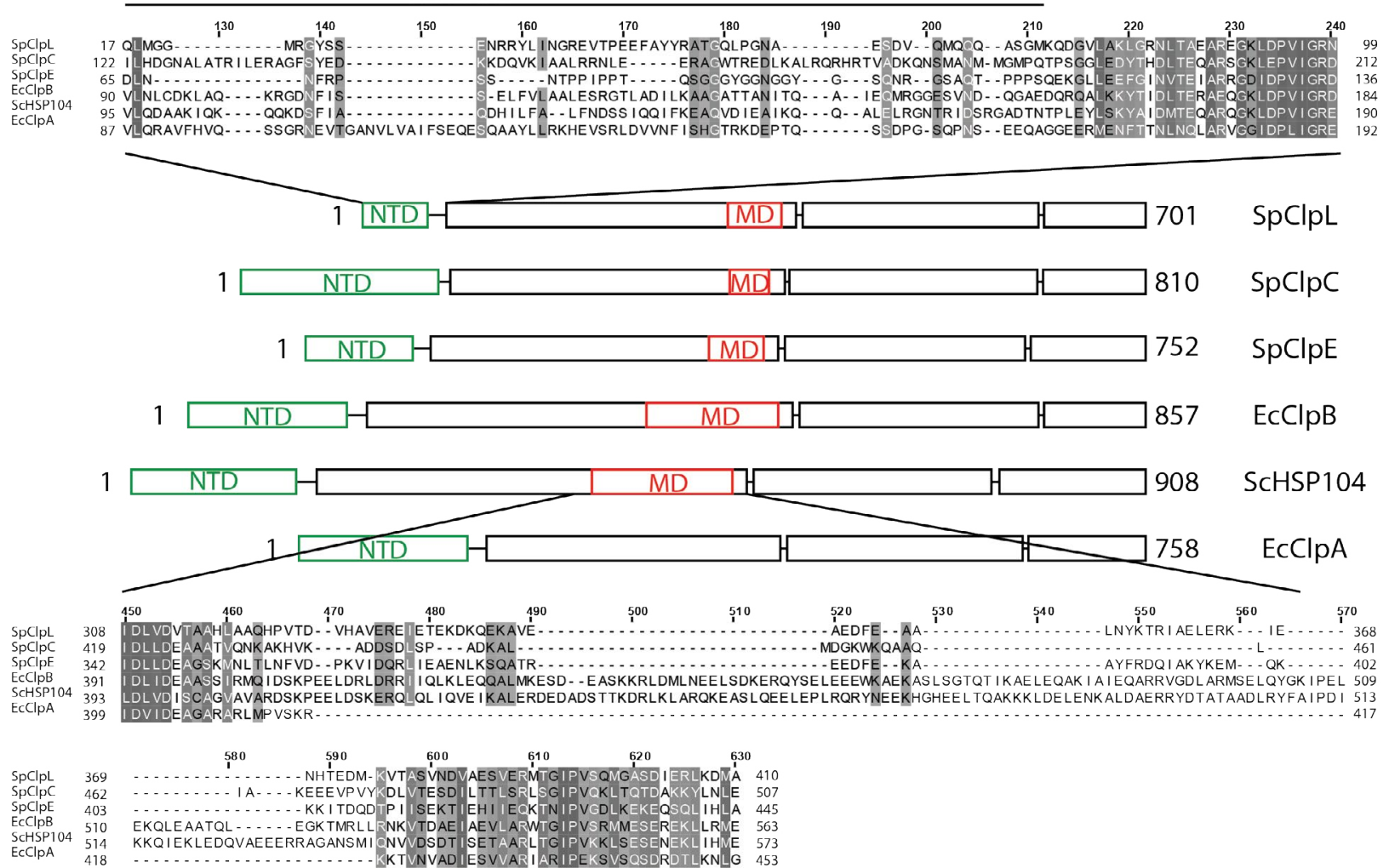

Figure 3: Comparison between the ClpL structure and that of other CIp/HSP100 proteins.

The protein sequence alignment of ClpL, ClpC, ClpE, ClpB, HSP104, and ClpA was generated using Clustal Omega (http://www.uniprot.org/align/). The N-terminal domain (NTD) and middle domain (MD) sequences are presented, as well as the R repeat region in the N-terminal domain. Conserved amino acids are highlighted in dark gray, and similar residues are highlighted in gray.

purification have been developed, but E. coli is currently used most widely. Owing to advances in recombinant technology, it is becoming more convenient to produce soluble proteins. However, despite many reports of successful heterologous protein expression, the use of bacterial co-chaperones, such as the DnaK and GroELS systems, to facilitate solubilization is not ideal. It is reported that during chaperone co-expression, undesired side effects, such as growth retardation and proteolysis, could give rise to reduced yield and reduced specific activity. Moreover, chaperone system per se is complex and sometimes induces protein aggregation. The lack of success in some cases does not suggest that this approach cannot be improved to produce functional proteins. For instance, pneumococcal ClpL is a very promising chaperone system 
that does not require co-chaperones, but the chaperone function of $\mathrm{ClpL}$ has not yet been tested in vivo. In addition, the study of chaperones may shed light on the virulence factors of pathogens, given that chaperones have been associated with the invasion, infection, and survival of virulent bacteria. For example, pneumococcal ClpL is induced by both cold shock and heat shock stress to protect bacteria from extreme conditions encountered by $S$. pneumoniae during infection. Thus, more extensive research on bacterial chaperons will undoubtedly provide us with useful information in regard to protein overexpression as well as bacterial virulence.

\section{Acknowledgement}

This work was supported by the Soonchunhyang University Research Fund to H.Y.K, and by the National Research Foundation to D.K.R (NRF-2015R1 A2 A1 A10052511).

\section{References}

1. Makrides SC (1996) Strategies for achieving high-level expression of genes in Escherichia coli. Microbiol Rev 60: 512-538.

2. Smith DB, Johnson KS (1988) Single-step purification of polypeptides expressed in Escherichia coli as fusions with glutathione S-transferase. Gene 67: $31-40$

3. di Guan C, Li P, Riggs PD, Inouye H (1988) Vectors that facilitate the expression and purification of foreign peptides in Escherichia coli by fusion to maltosebinding protein. Gene 67: 21-30.

4. Davis GD, Elisee C, Newham DM, Harrison RG (1999) New fusion protein systems designed to give soluble expression in Escherichia coli. Biotechnol Bioeng 65: 382-388.

5. Butt TR, Edavettal SC, Hall JP, Mattern MR (2005) SUMO fusion technology for difficult-to-express proteins. Protein Expr Purif 43: 1-9.

6. LaVallie ER, Lu Z, Diblasio-Smith EA, Collins-Racie LA, McCoy JM (2000) Thioredoxin as a fusion partner for production of soluble recombinant proteins in Escherichia coli. Methods Enzymol 326: 322-340.

7. Choi SI, Song HW, Moon JW, Seong BL (2001) Recombinant enterokinase light chain with affinity tag: expression from Saccharomyces cerevisiae and its utilities in fusion protein technology. Biotechnol Bioeng 75: 718-724.

8. Nagai K, Thøgersen HC (1984) Generation of beta-globin by sequence-specific proteolysis of a hybrid protein produced in Escherichia coli. Nature 309: 810-812.

9. Chang JY (1985) Thrombin specificity. Requirement for apolar amino acids adjacent to the thrombin cleavage site of polypeptide substrate. Eur J Biochem 151: $217-224$.

10. Chang JY, Alkan SS, Hilschmann N, Braun DG (1985) Thrombin specificity. Selective cleavage of antibody light chains at the joints of variable with joining regions and joining with constant regions. Eur J Biochem 151: 225-230.

11. Haun RS, Moss J (1992) Ligation-independent cloning of glutathione S-transferase fusion genes for expression in Escherichia coli. Gene 112: 37-43.

12. Daegelen P, Studier FW, Lenski RE, Cure S, Kim JF (2009) Tracing ancestors and relatives of Escherichia coli $\mathrm{B}$, and the derivation of B strains REL606 and BL21(DE3). J Mol Biol 394: 634-643.

13. Studier FW, Rosenberg AH, Dunn JJ, Dubendorff JW (1990) Use of T7 RNA polymerase to direct expression of cloned genes. Methods Enzymol 185: 60-89.

14. Carstens CP, Waesche A (1999) Codon bias-adjusted BL21 derivatives for protein expression. Strategies 12: 1.

15. Novy R, Drott D, Yaeger K, Mierendorf R (2001) Overcoming the codon bias of E. coli for enhanced protein expression. Innovations 12: 1-3.

16. Schein $\mathrm{CH}$ (1989) Production of Soluble Recombinant Proteins in Bacteria. Nat Biotech 7: 1141-1149.

17. Gopal GJ, Kumar A (2013) Strategies for the production of recombinant protein in Escherichia coli. Protein J 32: 419-425.

18. Hall D, Minton AP (2003) Macromolecular crowding: qualitative and semi quantitative successes, quantitative challenges. Biochim Biophys Acta 1649: 127-139.
19. Sasahara K, McPhie P, Minton AP (2003) Effect of dextran on protein stability and conformation attributed to macromolecular crowding. J Mol Biol 326: 1227-1237.

20. Despa F, Orgill DP, Lee RC (2005) Molecular crowding effects on protein stability. Ann N Y Acad Sci 1066: 54-66.

21. Despa F, Orgill DP, Lee RC (2005) Effects of crowding on the thermal stability of heterogeneous protein solutions. Ann Biomed Eng 33: 1125-1131.

22. Roman SG, Chebotareva NA, Eronina TB, Kleymenov SY, Makeeva VF, et al. (2011) Does the crowded cell-like environment reduce the chaperone-like activity of Ît-crystallin? Biochemistry 50: 10607-10623.

23. Martin J1 (2002) Requirement for GroEL/GroES-dependent protein folding under non-permissive conditions of macromolecular crowding. Biochemistry 41: 5050-5055.

24. Chow KC, Tung WL (1998) Overexpression of dnaK/dnaJ and groEL confers freeze tolerance to Escherichia coli. Biochem Biophys Res Commun 253: 502-505.

25. Rosenzweig R, Moradi S, Zarrine-Afsar A, Glover JR, Kay LE (2013) Unraveling the mechanism of protein disaggregation through a ClpB-DnaK interaction. Science 339: 1080-1083.

26. Hartl FU, Hayer-Hartl M (2002) Molecular chaperones in the cytosol: from nascent chain to folded protein. Science 295: 1852-1858.

27. Erbse A, Yifrach O, Jones S, Lund PA (1999) Chaperone activity of a chimeric GroEL protein that can exist in a single or double ring form. J Biol Chem 274 20351-20357.

28. Chatellier J, Hill F, Foster NW, Goloubinoff P, Fersht AR (2000) From minichaperone to GroEL 3: properties of an active single-ring mutant of GroEL. J Mol Biol 304: 897-910.

29. Ogura T, Wilkinson AJ (2001) AAA+ superfamily ATPases: common structure-diverse function. Genes Cells 6: 575-597.

30. Doyle SM, Wickner S (2009) Hsp104 and ClpB: protein disaggregating machines. Trends Biochem Sci 34: 40-48.

31. Park SS, Kwon HY, Tran TD, Choi MH, Jung SH, et al. (2015) ClpL is a chaperone without auxiliary factors. FEBS J 282: 1352-1367.

32. Kim YI, Levchenko I, Fraczkowska K, Woodruff RV, Sauer RT, et al (2001) Molecular determinants of complex formation between Clp/Hsp100 ATPases and the ClpP peptidase. Nat Struct Biol 8: 230-233.

33. Seyffer F, Kummer E, Oguchi Y, Winkler J, Kumar M, et al. (2012) Hsp70 proteins bind Hsp100 regulatory $M$ domains to activate $A A A+$ disaggregase at aggregate surfaces. Nat Struct Mol Biol 19: 1347-1355.

34. Carroni M, Kummer E, Oguchi Y, Wendler P, Clare DK, et al. (2014) Head-to-tail interactions of the coiled-coil domains regulate ClpB activity and cooperation with Hsp70 in protein disaggregation. Elife 3: e02481.

35. Mogk A, Schlieker C, Strub C, Rist W, Weibezahn J, et al. (2003) Roles of individual domains and conserved motifs of the AAA+ chaperone ClpB in oligomerization, ATP hydrolysis, and chaperone activity. J Biol Chem 278 : 17615-17624.

36. Aguado A, Fernández-Higuero JA, Cabrera Y, Moro F, Muga A1 (2015) ClpB dynamics is driven by its ATPase cycle and regulated by the DnaK system and substrate proteins. Biochem J 466: 561-570.

37. Kedzierska S, Akoev V, Barnett ME, Zolkiewski M (2003) Structure and function of the middle domain of ClpB from Escherichia coli. Biochemistry 42: 1424214248.

38. Haslberger T, Weibezahn J, Zahn R, Lee S, Tsai FT, et al. (2007) M domains couple the ClpB threading motor with the DnaK chaperone activity. Mol Cell 25: $247-260$

39. Watanabe YH, Nakazaki Y, Suno R, Yoshida M (2009) Stability of the two wings of the coiled-coil domain of $\mathrm{ClpB}$ chaperone is critical for its disaggregation activity. Biochem J 421: 71-77.

40. Wendler P, Shorter J, Plisson C, Cashikar AG, Lindquist S, et al. (2007) Atypical $A A A+$ subunit packing creates an expanded cavity for disaggregation by the protein-remodeling factor Hsp104. Cell 131: 1366-1377.

41. Doyle SM, Hoskins JR, Wickner S (2007) Collaboration between the ClpB $\mathrm{AAA}+$ remodeling protein and the DnaK chaperone system. Proc Natl Acad Sc USA 104: 11138-11144. 
Citation: Kwon HY, Park SS, Zia MF, Rhee DK (2016) Protein Expression via the Molecular Chaperone ClpL. J Microb Biochem Technol 8: 076-082. doi: 10.4172/1948-5948.1000266

42. Baker TA, Sauer RT (2006) ATP-dependent proteases of bacteria: recognition logic and operating principles. Trends Biochem Sci 31: 647-653.

43. Schlothauer T, Mogk A, Dougan DA, Bukau B, Turgay K (2003) MecA, an adaptor protein necessary for ClpC chaperone activity. Proc Natl Acad Sci U S A 100: 2306-2311.

44. Wickner S, Gottesman S, Skowyra D, Hoskins J, McKenney K, et al. (1994) A molecular chaperone, ClpA, functions like DnaK and DnaJ. Proc Natl Acad Sci U S A 91: 12218-12222.

45. Robertson GT, Ng WL, Gilmour R, Winkler ME (2003) Essentiality of clpX, but not clpP, clpL, clpC, or clpE, in Streptococcus pneumoniae R6. J Bacteriol 185: 2961-2966.

46. Robertson GT, Ng WL, Foley J, Gilmour R, Winkler ME (2002) Global transcriptional analysis of clpP mutations of type 2 Streptococcus pneumoniae and their effects on physiology and virulence. J Bacteriol 184: 3508-3520.

47. Charpentier E, Novak R, Tuomanen E (2000) Regulation of growth inhibition at high temperature, autolysis, transformation and adherence in Streptococcus pneumoniae by clpC. Mol Microbiol 37: 717-726.

48. Zhang Q, Xu SX, Wang H, Xu WC, Zhang XM, et al. (2009) Contribution of ClpE to virulence of Streptococcus pneumoniae. Can J Microbiol 55: 11871194.

49. Kwon HY, Kim SW, Choi MH, Ogunniyi AD, Paton JC, et al. (2003) Effect of heat shock and mutations in ClpL and ClpP on virulence gene expression in Streptococcus pneumoniae. Infect Immun 71: 3757-3765.

50. Tran TD, Kwon HY, Kim EH, Kim KW, Briles DE, et al. (2011) Decrease in penicillin susceptibility due to heat shock protein ClpL in Streptococcus pneumoniae. Antimicrob Agents Chemother 55: 2714-2728.

51. Tao L, Biswas I (2013) ClpL is required for folding of CtsR in Streptococcus mutans. J Bacteriol 195: 576-584.
52. Conlon BP, Nakayasu ES, Fleck LE, LaFleur MD, Isabella VM, et al. (2013) Activated ClpP kills persisters and eradicates a chronic biofilm infection. Nature 503: $365-370$

53. Nguyen CT, Le NT, Tran TD, Kim EH, Park SS, et al. (2014) Streptococcus pneumoniae ClpL modulates adherence to A549 human lung cells through Rap1/Rac1 activation. Infect Immun 82: 3802-3810.

54. Diamant S, Azem A, Weiss C, Goloubinoff P (1995) Increased efficiency of GroE-assisted protein folding by manganese ions. J Biol Chem 270: 2838728391.

55. El Bakkouri M, Pow A, Mulichak A, Cheung KL, Artz JD, et al. (2010) The Clp chaperones and proteases of the human malaria parasite Plasmodium falciparum. J Mol Biol 404: 456-477.

56. Dougan DA, Reid BG, Horwich AL, Bukau B (2002) ClpS, a substrate modulator of the ClpAP machine. Mol Cell 9: 673-683.

57. Singh SK, Rozycki J, Ortega J, Ishikawa T, Lo J, et al. (2001) Functiona domains of the ClpA and ClpX molecular chaperones identified by limited proteolysis and deletion analysis. J Biol Chem 276: 29420-29429.

58. Kirstein J, Schlothauer T, Dougan DA, Lilie H, Tischendorf G, et al. (2006) Adaptor protein controlled oligomerization activates the AAA+ protein ClpC. EMBO J 25: 1481-1491.

59. Tek V, Zolkiewski M (2002) Stability and interactions of the amino-terminal domain of ClpB from Escherichia coli. Protein Sci 11: 1192-1198.

60. Barnett ME, Nagy M, Kedzierska S, Zolkiewski M (2005) The amino-terminal domain of ClpB supports binding to strongly aggregated proteins. J Biol Chem 280: 34940-34945.

61. Lee S, Sowa ME, Choi JM, Tsai FT (2004) The ClpB/Hsp104 molecular chaperone-a protein disaggregating machine. J Struct Biol 146: 99-105. 